\title{
QUASI-SIMILARITY OF WEAK CONTRACTIONS
}

\author{
PEI YUAN WU
}

\begin{abstract}
Let $T$ be a completely nonunitary (c.n.u.) weak contraction (in the sense of Sz.-Nagy and Foias). We show that $T$ is quasi-similar to the direct sum of its $C_{0}$ part and $C_{11}$ part. As a corollary, two c.n.u. weak contractions are quasi-similar to each other if and only if their $C_{0}$ parts and $C_{11}$ parts are quasi-similar to each other, respectively. We also completely determine when c.n.u. weak contractions and $C_{0}$ contractions are quasi-similar to normal operators.
\end{abstract}

Recall that a contraction $T$ on the Hilbert space $H$ is called a weak contraction if its spectrum $\sigma(T)$ does not fill the open unit disc $D$ and $1-T^{*} T$ is of finite trace. Contained in this class are all contractions $T$ with finite defect index $d_{T} \equiv \operatorname{dim} \operatorname{rank}\left(1-T^{*} T\right)^{1 / 2}$ and with $\sigma(T) \neq \bar{D}$ (cf. [9, p. 323]).

Assume that $T$ is a weak contraction which is also completely nonunitary (c.n.u.), that is, $T$ has no nontrivial reducing subspace on which $T$ is a unitary operator. For such a contraction, Sz.-Nagy and Foiaş obtained a $C_{0}-C_{11}$ decomposition and then found a variety of invariant subspaces which furnish its spectral decomposition (cf. [9, Chapter VIII]). In this note we are going to supplement other interesting properties of such contractions. We show that a c.n.u. weak contraction is quasi-similar to the direct sum of its $C_{0}$ part and $C_{11}$ part. Although the proof is not difficult, some of its interesting applications justify the elaboration here. An immediate corollary is that two such contractions are quasi-similar to each other if and only if their $C_{0}$ parts are quasi-similar and their $C_{11}$ parts are quasi-similar to each other. This is, in turn, used to show that two quasi-similar weak contractions have equal spectra. Another interesting consequence is that a c.n.u. weak contraction is quasi-similar to a normal operator if and only if its $C_{0}$ part is. The latter can be shown to be equivalent to the condition that its minimal function is a Blaschke product with simple zeros, thus completely settling the question when a c.n.u. weak contraction is quasi-similar to a normal operator.

Before we start to prove our main theorem, we provide some background work for our notations and terminology. The main reference is [9].

Presented to the Society September 9, 1976; received by the editors October 1, 1976.

AMS (MOS) subject classifications (1970). Primary 47A45.

$K e y$ words and phrases. Completely nonunitary weak contractions, quasi-similarity, $C_{0}$ parts, $C_{11}$ parts, normal operators.

'This research was done while the author was visiting Indiana University during the summer of 1976.

(1) American Mathematical Society 1978 
Let $T$ be an arbitrary contraction on $H$. Let $H_{0}=\left\{h \in H: T^{n} h \rightarrow 0\right\}$, $H_{0}^{\prime}=\left\{h \in H: T^{* n} h \rightarrow 0\right\}, H_{1}^{\prime}=H \ominus H_{0}$ and $H_{1}=H \ominus H_{0}^{\prime}$. Note that $H_{0}$ and $H_{0}^{\prime}$ are invariant for $T$ and $T^{*}$, respectively. Consider the triangulations of $T$ with respect to the orthogonal decompositions $H=H_{0} \oplus H_{1}^{\prime}$ and $H=H_{1} \oplus H_{0}^{\prime}$ :

$$
T=\left[\begin{array}{ll}
T_{0} & X \\
0 & T_{1}^{\prime}
\end{array}\right] \text { and } T=\left[\begin{array}{ll}
T_{1} & Y \\
0 & T_{0}^{\prime}
\end{array}\right] .
$$

The triangulations are of type

$$
\left[\begin{array}{ll}
C_{0} & * \\
0 & C_{1 .}
\end{array}\right] \text { and }\left[\begin{array}{ll}
C_{.1} & * \\
0 & C_{.0}
\end{array}\right] \text {, }
$$

respectively (cf. [9, p. 73]). Recall that a contraction $T$ is of class $C_{0}$. (resp. $C_{.0}$ ) if $T^{n} h \rightarrow 0$ (resp. $\mathrm{T}^{* n} h \rightarrow 0$ ) as $n \rightarrow \infty$ for all $h$ and $T$ is of class $C_{1}$. (resp. $C_{\text {.1) }}$ ) if $T^{n} h \nrightarrow 0$ (resp. $T^{* n} h \nrightarrow 0$ ) as $n \rightarrow \infty$ for all $h \neq 0$. T is of class $C_{00}$ if $T \in C_{0} \cap \cap C_{.0}$ and of class $C_{11}$ if $T \in C_{1} \cap \cap C_{\text {. }}$. A c.n.u. contraction $T$ is said to be of class $C_{0}$ if there exists a nonzero function $u \in H^{\infty}$ such that $u(T)=0$. In this case we can choose $u$ to be a minimal inner function in the sense that $u$ is an inner function such that $u(T)=0$ and $u$ divides (in $H^{\infty}$ ) every other function $v \in H^{\infty}$ for which $v(T)=0$. Such a function is called a minimal function for $T$ and is denoted by $m_{T}$. If $T$ is a c.n.u. weak contraction, then in the previous triangulations $T_{0}$ is of class $C_{0}$ and $T_{1}$ is of class $C_{11}$, called the $C_{0}$ part and the $C_{11}$ part of $T$ (cf. [9, p. 331]). Note that in this case we have $H_{0} \vee H_{1}=H$ and $H_{0} \cap H_{1}=\{0\}$ (cf. [9, p. 332]). For arbitrary operators $T_{1}, T_{2}$ on $H_{1}, H_{2}$, respectively, $T_{1} \prec T_{2}$ denotes that $T_{1}$ is a quasi-affine transform of $T_{2}$, that is, there exists a linear one-to-one and continuous transformation $S$ from $H_{1}$ onto a dense linear manifold in $H_{2}$ (called quasi-affinity) such that $S T_{1}=T_{2} S . T_{1}$ and $T_{2}$ are quasi-similar if $T_{1} \prec T_{2}$ and $T_{2} \prec T_{1}$.

Our main theorem is the following:

THEOREM 1. Let $T$ be a c.n.u. weak contraction on H. Let $T_{0}$ and $T_{1}$ be the $C_{0}$ part and $C_{11}$ part of $T$. Then $T$ is quasi-similar to $T_{0} \oplus T_{1}$.

Proof. Let $S: H_{0} \oplus H_{1} \rightarrow H$ be defined by $S\left(h_{0} \oplus h_{1}\right)=h_{0}+h_{1}$. Certainly $T$ is a continuous linear transformation. Since $H_{0} \vee H_{1}=H$ and $H_{0} \cap H_{1}=\{0\}$, it is easily seen that $S$ is a quasi-affinity such that $S\left(T_{0} \oplus\right.$ $\left.T_{1}\right)=T S$. Thus $T_{0} \oplus T_{1} \prec T$. Note that $T^{*}$ is also a c.n.u. weak contraction and $T_{0}^{\prime *}$ and $T_{1}^{\prime *}$ are the $C_{0}$ and $C_{11}$ parts of $T^{*}$ (cf. [9, p. 332]). As above, we have $T_{0}^{\prime *} \oplus T_{1}^{\prime *} \prec T^{*}$. Hence $T \prec T_{0}^{\prime} \oplus T_{1}^{\prime}$, and $T_{0} \oplus T_{1} \prec T \prec T_{0}^{\prime} \oplus T_{1}^{\prime}$. Let $V$ be the quasi-affinity from $H_{0} \oplus H_{1}$ to $H_{0}^{\prime} \oplus H_{1}^{\prime}$ such that $V\left(T_{0} \oplus T_{1}\right)$ $=\left(T_{0}^{\prime} \oplus T_{1}^{\prime}\right) V$. Since $T_{0}$ and $T_{1}^{\prime}$ are of class $C_{0}$. and $C_{1}$, respectively, it is easily seen that $V H_{0} \subseteq H_{0}^{\prime}$. Say,

$$
V=\left[\begin{array}{ll}
V_{0} & Z \\
0 & V_{1}
\end{array}\right]
$$


is the corresponding triangulation. An easy calculation shows that $Z T_{1}=$ $T_{0}^{\prime} Z$. Since $T_{1}$ is of class $C_{11}$ and $T_{0}^{\prime}$ is of class $C_{00}$, we must have $Z=0$ (cf. [4, Lemma 4.4]). Thus $V_{0}$ and $V_{1}$ are quasi-affinities satisfying $V_{0} T_{0}=T_{0}^{\prime} V_{0}$ and $V_{1} T_{1}=T_{1}^{\prime} V_{1}$. Hence $T_{0} \prec T_{0}^{\prime}$ and $T_{1} \prec T_{1}^{\prime}$. It follows from the uniqueness of the Jordan model for $C_{0}$ contractions that $T_{0}$ and $T_{0}^{\prime}$ are quasi-similar to each other (cf. [2]). To show that $T_{1}$ is quasi-similar to $T_{1}^{\prime}$, note that $T_{1}$ and $T_{1}^{\prime}$, being $C_{11}$ contractions, are quasi-similar to unitary operators, say $U_{1}$ and $U_{1}^{\prime}$, respectively. We have $U_{1} \prec U_{1}^{\prime}$. By a theorem of Douglas [5], $U_{1}$ and $U_{1}^{\prime}$ are unitarily equivalent. Hence $T_{1}$ is quasi-similar to $T_{1}^{\prime}$, and $T$ is quasi-similar to $T_{0} \oplus T_{1}$.

An immediate corollary of Theorem 1 is

Corollary 1. Let $T_{1}$ and $T_{2}$ be c.n.u. weak contractions. Then $T_{1}$ and $T_{2}$ are quasi-similar to each other if and only if their $C_{0}$ parts are quasi-similar and their $C_{11}$ parts are quasi-similar to each other.

Proof. The sufficiency follows immediately from Theorem 1 . The necessity can be proved by a similar argument as in Theorem 1 .

In particular, for c.n.u. contractions with scalar-valued characteristic functions, we have

COROLlaRy 2. For $j=1,2$, let $T_{j}$ be a c.n.u. contraction with the scalar-valued characteristic function $\psi_{j} \not \equiv 0$. Let $\psi_{j}=\psi_{j i} \psi_{j e}$ be the canonical factorization into the product of its inner part $\psi_{j i}$ and outer part $\psi_{j e}$, and let $E_{j}=\left\{e^{i t}\right.$ : $\left.\left|\psi_{j}\left(e^{i t}\right)\right|<1\right\}$. Let

$$
T_{j}=\left(\begin{array}{ll}
T_{j 1} & X_{j} \\
0 & T_{j 2}
\end{array}\right)
$$

be the triangulation of type

$$
\left[\begin{array}{ll}
C_{.1} & * \\
0 & C_{.0}
\end{array}\right], \quad j=1,2 .
$$

Then the following are equivalent:

(i) $T_{1}$ is quasi-similar to $T_{2}$;

(ii) $T_{11}$ is quasi-similar to $T_{21}$ and $T_{12}$ is unitarily equivalent to $T_{22}$;

(iii) $\psi_{1 i}=\psi_{2 i}$ and $E_{1}$ and $E_{2}$ differ by a set of zero Lebesgue measure.

Proof. Since $T_{1}$ and $T_{2}$ are c.n.u. weak contractions, the equivalence of (i) and (ii) follows from Corollary 1 . Note that $T_{j 1}$ is quasi-similar to the multiplication by $e^{i t}$ on the space $L^{2}\left(E_{j}\right)$ and $T_{j 2}$ is unitarily equivalent to the compression of the shift $S\left(\psi_{j i}\right)$ on $H^{2} \Theta \psi_{j i} H^{2}, j=1,2$. Thus the equivalence of (ii) and (iii) follows immediately.

The equivalence of (i) and (iii) in Corollary 2 is compatible with the result of Kriete [7] that $T_{1}$ is similar to $T_{2}$ if and only if $\psi_{1} / \psi_{2}, \psi_{2} / \psi_{1} \in H^{\infty}$ and $E_{1}$ and $E_{2}$ differ by a set of zero Lebesgue measure.

Corollary 3. Let $T_{1}$ and $T_{2}$ be c.n.u. weak contractions. If $T_{1}$ and $T_{2}$ are 
quasi-similar to each other, then $\sigma\left(T_{1}\right)=\sigma\left(T_{2}\right)$.

Proof. For $j=1,2$, let $T_{j 0}$ and $T_{j 1}$ be the $C_{0}$ part and $C_{11}$ part of $T_{j}$. By Corollary $1, T_{10}$ and $T_{11}$ are quasi-similar to $T_{20}$ and $T_{21}$, respectively. Since the spectrum of a $C_{0}$. contraction is completely determined by its minimal function [9, p. 126], and $T_{10}$ and $T_{20}$ have the same minimal function, we have $\sigma\left(T_{10}\right)=\sigma\left(T_{20}\right)$.

To show that $\sigma\left(T_{11}\right)=\sigma\left(T_{21}\right)$, let $U_{j}$ be the residual part of the minimal unitary dilation of $T_{j 1}, j=1,2$ (cf. [9, p. 61]). Note that $T_{j 1}$ is quasi-similar to $U_{j}$ and $\sigma\left(T_{j 1}\right)$ lies entirely on the unit circle (cf. [9, pp. 75, 328]). It follows that $\sigma\left(T_{j 1}\right)=\sigma\left(U_{j}\right)$ (cf. [9, pp. 311-312]). By Douglas' theorem [5], $U_{1}$ and $U_{2}$ are quasi-similar implies they are unitarily equivalent. Thus $\sigma\left(T_{11}\right)=\sigma\left(U_{1}\right)=$ $\sigma\left(U_{2}\right)=\sigma\left(T_{21}\right)$. Since $\sigma\left(T_{j}\right)=\sigma\left(T_{j 0}\right) \cup \sigma\left(T_{j 1}\right)\left[9\right.$, p. 332], we have $\sigma\left(T_{1}\right)=$ $\sigma\left(T_{2}\right)$, completing the proof.

We remark that the proof can be modified to show that quasi-similar weak contractions (not necessarily c.n.u.) have equal spectra. This result is not new. It also follows from the facts that weak contractions are decomposable [6] and quasi-similar decomposable operators have equal spectra [3]. However, our proof seems more direct.

In the remaining part of this note we are concerned with the question when a c.n.u. weak contraction is quasi-similar to a normal operator. The next theorem reduces the problem to the $C_{0}$ part of the c.n.u. weak contraction.

THEOREM 2. Let $T$ be a c.n.u. weak contraction on $H$. Let

$$
T=\left[\begin{array}{ll}
T_{0} & X \\
0 & T_{1}^{\prime}
\end{array}\right]
$$

be the triangulation of type

$$
\left[\begin{array}{ll}
C_{0} & * \\
0 & C_{1}
\end{array}\right]
$$

on the (orthogonal) decomposition $H=H_{0} \oplus H_{1}^{\prime}$. Then $T$ is quasi-similar to a normal operator if and only if $T_{0}$ is.

Proof. The sufficiency follows trivially from Theorem 1. To prove the necessity, we may assume that $T$ is quasi-similar to a normal operator $N$ on the space $K$ with $\|N\| \leqslant\|T\| \leqslant 1$ (cf. [1, Proof of the sufficiency part of Theorem]). Let $K=K_{1} \oplus K_{2}$ be the direct sum of reducing subspaces for $N$ such that $N_{1} \equiv N \mid K_{1}$ is c.n.u. and $N_{2} \equiv N \mid K_{2}$ is unitary. Let $S$ be the quasi-affinity from $H$ to $K$ such that $S T=N S$. Since $T_{0}$ is of class $C_{0}$ and $N_{2}$ is of class $C_{11}$, it is easily seen that $S H_{0} \subseteq K_{1}$. Note that $\overline{S H}_{0}$ is an invariant subspace for $N_{1}$. Let $N_{1}^{\prime}=N_{1} \mid \overline{S H}_{0}$. Then $S_{1} \equiv S \mid H_{0}$ is a quasi-affinity from $H_{0}$ to $\overline{S H}_{0}$ satisfying $S_{1} T_{0}=N_{1}^{\prime} S_{1}$. Since $T_{0}$ is of class $C_{0}$, so is $N_{1}^{\prime}$ (cf. [9, p. 125]). By the uniqueness of the Jordan model for $C_{0}$ contractions, we have $T_{0}$ is quasi-similar to $N_{1}^{\prime}$ (cf. [2]). Since $N_{1}^{\prime}$ is subnormal and $\sigma\left(N_{1}^{\prime}\right)$ has planar 
area zero (cf. [9, p. 126]), it follows from Putnam's theorem [8] that $N_{1}^{\prime}$ is normal. This completes the proof.

Notice that Theorem 2 is compatible with the result that $T$ is similar to a normal operator if and only if $T_{0}$ is similar to a normal operator and $T_{1}^{\prime}$ is similar to a unitary operator. This is true even for an arbitrary c.n.u. contraction (cf. [10, Theorem 3]).

Since the $C_{0}$ part of a c.n.u. weak contraction is a $C_{0}$ contraction, the next theorem furnishes the complete solution to the previously posed question.

THeOREM 3. Let $T$ be a $C_{0}$ contraction on the space $H$ with the minimal function $m_{T}$. Then $T$ is quasi-similar to a normal operator if and only if $m_{T}$ is a Blaschke product with simple zeros.

Proof. Necessity. Let $T$ be quasi-similar to the normal operator $N$ on the space $K$ and let $S$ be the quasi-affinity from $H$ to $K$ such that $S T=N S$. As before we may assume that $\|N\| \leqslant\|T\| \leqslant 1$ (cf. [1]). Now we show that $N$ must be c.n.u. Indeed, for any $k \in K$ and $\varepsilon>0$, let $h \in H$ be such that $\|k-S h\|<\varepsilon$. Since $S T^{n} h=N^{n} S h \rightarrow 0$ as $n \rightarrow \infty$, we have $\left\|N^{n} S h\right\|<\varepsilon$ for all $n \geqslant N_{0}$. Hence

$$
\begin{aligned}
\left\|N^{n} k\right\| & \leqslant\left\|N^{n} k-N^{n} S h\right\|+\left\|N^{n} S h\right\| \leqslant\|N\|^{n}\|k-S h\|+\left\|N^{n} S h\right\| \\
& <\varepsilon+\varepsilon=2 \varepsilon \text { for all } n \geqslant N_{0} .
\end{aligned}
$$

This shows that $N^{n} k \rightarrow 0$ for all $k \in K$ and hence $N$ is c.n.u. Since $N$ is quasi-similar to a $C_{0}$ contraction, $N$ is also a $C_{0}$ contraction with the same minimal function $m_{N}=m_{T}$ (cf. [9, p. 125]). Let $m_{T}=B s$, where

$$
B(\lambda)=\prod_{i} \frac{\bar{\lambda}_{i}}{\left|\lambda_{i}\right|}\left(\frac{\lambda_{i}-\lambda}{1-\bar{\lambda}_{i} \lambda}\right)^{n_{i}}
$$

is a Blaschke product and $s$ is a singular function. Note that $\lambda_{i}$ is a characteristic value of $N$ with index $n_{i}$ (cf. [9, p. 135]). Since $N$ is a normal operator, $n_{i}=1$ for all $i$. Let $K_{i}$ be the corresponding eigenspace. Then $\bigvee_{i} K_{i}$ reduces $N$ and the normal operator $N_{1} \equiv N \mid\left(\bigvee_{i} K_{i}\right)^{\perp}$ has no eigenvalue. Hence the minimal function of the $C_{0}$ contraction $N_{1}$ must be $s$ (cf. [9. p. 129]). It follows that $\sigma\left(N_{1}\right)$ is contained in the unit circle, and thus $N_{1}$ is a unitary operator. Since $N$ is c.n.u., we must have $\left(\bigvee_{i} K_{i}\right)^{\perp}=\{0\}$ and $K=$ $\bigvee_{i} K_{i}$. Hence $m_{T}=B$ is a Blaschke product with simple zeros (cf. [9, p. 135]).

Sufficiency. Assume that $m_{T}$ is a Blaschke product with simple zeros, say,

$$
m_{T}(\lambda)=\prod_{i} \frac{\bar{\lambda}_{i}}{\left|\lambda_{i}\right|} \frac{\lambda_{i}-\lambda}{1-\bar{\lambda}_{i} \lambda}
$$

where the distinct $\lambda_{i}$ 's satisfy $\left|\lambda_{i}\right|<1$ and $\Sigma_{i}\left(1-\left|\lambda_{i}\right|\right)<\infty$. For each $i$ let $H_{i}=\left\{h \in H:\left(T-\lambda_{i}\right) h=0\right\}$. Then $T \mid H_{i}$ is a normal operator and the system $\left\{H_{i}\right\}_{i=1}^{\infty}$ of invariant subspaces satisfies

$$
H=H_{i}+\bigvee_{j \neq i} H_{j} \text { for each } i, \text { and } \bigcap_{i}\left(\bigvee_{j>i} H_{j}\right)=\{0\}
$$


(cf. [9, pp. 135, 131]). That is, $\left\{H_{i}\right\}_{i=1}^{\infty}$ is a basic system of invariant subspaces for $T$. By a result of Apostol [1], $T$ is quasi-similar to a normal operator, completing the proof.

\section{REFERENCES}

1. C. Apostol, Operators quasi-similar to a normal operator, Proc. Amer. Math. Soc. 53 (1975), 104-106.

2. H. Bercovici, C. Foias and B. Sz.-Nagy, Compléments à l'étude des opérateurs de classe co. III, Acta Sci. Math. (Szeged) 37 (1975), 313-322.

3. I. Colojoară and C. Foias, Theory of generalized spectral operators, Gordon and Breach, New York, 1968.

4. J. B. Conway and $\mathrm{P}$. Y. Wu, The splitting of $\mathbb{Q}\left(T_{1} \oplus T_{2}\right)$ and related questions, Indiana Univ. Math. J. 26 (1977), 41-56.

5. R. G. Douglas, On the operator equation $S^{*} X T=X$ and related topics, Acta Sci. Math. (Szeged) 30 (1969), 19-32.

6. A. A. Jafarian, Weak contractions of Sz.-Nagy and Foias are decomposable, Rev. Roumaine Math. Pures Appl. 22 (1977), 489-497.

7. T. L. Kriete, III, Similarity of canonical models, Bull. Amer. Math. Soc. 76 (1970), 326-330.

8. C. R. Putnam, An inequality for the area of hyponormal spectra, Math. Z. 116 (1970), 323-330.

9. B. Sz.-Nagy and C. Foias, Harmonic analysis of operators on Hilbert space, Akadémiai Kiado, Budapest, 1970.

10. P. Y. Wu, On nonorthogonal decompositions of certain contractions, Acta Sci. Math. (Szeged) 37 (1975), 301-306.

Department of Applied Mathematics, National Chiao Tung University, Hsinchu, TaIwan, Republic OF China 